\section{What will we learn from}

\section{ITER?}

\author{
Jo Lister ${ }^{1}$ and Henri Weisen ${ }^{2}$ \\ ${ }^{1}$ Centre de Recherches en Physique des Plasmas, CRPP-EPFL, \\ ${ }^{2}$ Ecole Polytechnique Fédérale de Lausanne, 1015 Lausanne, \\ Switzerland
}

A $t$ the time of writing, we are once again at the threshold of an international agreement to go ahead with the construction of the ITER (International Thermonuclear Experimental Reactor) project, uniting the research efforts of Europe, Japan, China, Korea, Russia and the USA. It is very much the hope that at the time of reading, preparations at the construction site, whether in Europe or Japan, will be well underway. The project is extremely ambitious and carries a significant construction cost, roughly $500 \mathrm{M} €$ per year over 10 years of construction. If built in Europe, the likely domestic cost will be $250 \mathrm{M} €$ per year to the European taxpayer, including Switzerland. Although this is of course very large for a scientific research project, it still only represents the sum of $2.5 €$, or the average price of a small beer in Europe for each taxpayer every year, a much lower cost than many marketing projects.

Why spend so much? What will we learn from ITER? These questions have been raised in some academic circles, contesting the size of such an investment in a scientific experiment. We consider it judicious to attempt a reply, addressed to the general physics research community, explaining what will be achieved and especially what lessons will be learned. Is ITER a project in the basic sciences lineage, searching ultimate truths about the nature of the world, or about the origins of our universe? The answer is "No". Such questions have an essential purity about them, a purity that has always appealed, both to the initiated and to the uninitiated, as representing a noble cause, a scientific golden fleece. The world's largest accelerators have always received relatively constant support in Europe, in spite of the difficulty the public at large has to grasp the significance of the results obtained, or even understand the questions being addressed.

ITER is nonetheless a noble cause, even though its main motivation stems from our increasingly urgent quest for sustainable energy. The nobility resides equally in the physical understanding to be acquired of the complexity of plasmas and in the technical challenges to be met. The requirements for controlled nuclear fusion are potent drivers for advances in physics and technology. This quest has also brought a harvest of fundamental knowledge in physics, in such complex areas as turbulence, magnetohydrodynamics and even material sciences, with implications for apparently unrelated areas such as astrophysics, space physics and industrial plasmas, spawning applications ranging from plasma processing to space propulsion systems, the development of novel materials and superconductors. The ITER project is set to take this endeavour a major step further into uncharted territory.

In the following we shall try to remind the reader of the "why ITER?" with a brief introduction and then recall the present state of our research into controlled fusion using magnetic confinement of plasmas. The status today is, simply stated, that we believe that our long-term vision can become reality, but we need to make a leap forwards to demonstrate this. ITER is the long-awaited step needed to take fusion out from the present large laboratory experiment in the direction of a full power station. We shall see that our question "why ITER?" leads to a simple answer "to establish whether our vision can become real".

\section{Basics of controlled fusion}

A previous special issue of Europhysics News (Vol. 29, 6, Nov/Dec 1998) contained an excellent set of articles on controlled fusion research, so our introduction here is brief and the motivated reader is encouraged to return to this special issue for more details.

The construction of any nucleus out of its neutron and proton building blocks leaves a total mass that is slightly less than the mass of its constituents, making the nucleus more or less energetically stable. This binding energy is lowest for very large nuclei, therefore only weakly bound, and for very small nuclei, therefore also very weakly bound. This is best seen in the wellknown binding energy, or missing mass curve. Breaking apart a large nucleus leaves two more stable nuclei and the difference in binding energy is released as kinetic energy of the resulting nuclear fractions, the process known as nuclear fission. Combining two small nuclei also leads to a more stable nucleus and again the difference in binding energy is released as kinetic energy of the final state products, the process known as nuclear fusion. The energy released in both classes of reaction is enormous $(\mathrm{MeV})$ compared with molecular or atomic energy changes $(\mathrm{eV})$.

Our understanding of nuclear fusion and of nuclear fission emerged in the 1930's. Although fission reactors started delivering power during the following decade, it's only 6 decades later that a modest 16MW of fusion power were produced for a second by the JET (Joint European Torus) tokamak sited at Culham in the UK [1]. Why is fusion power generation so much more difficult?

The underlying reason lies in a fundamental difference between fission and fusion. The problem of running a fission reactor is getting it to go slowly, releasing the energy stored in the reactor fuel ( $200 \mathrm{GJ})$ over a period of 2 years rather than in a fraction of a second. The fission reaction occurs as a chain reaction, the neutrons released from one reaction triggering the following reactions. Chain reactions can run away, deliberately in a weapon and accidentally in a power plant. For the fusion reaction, there is

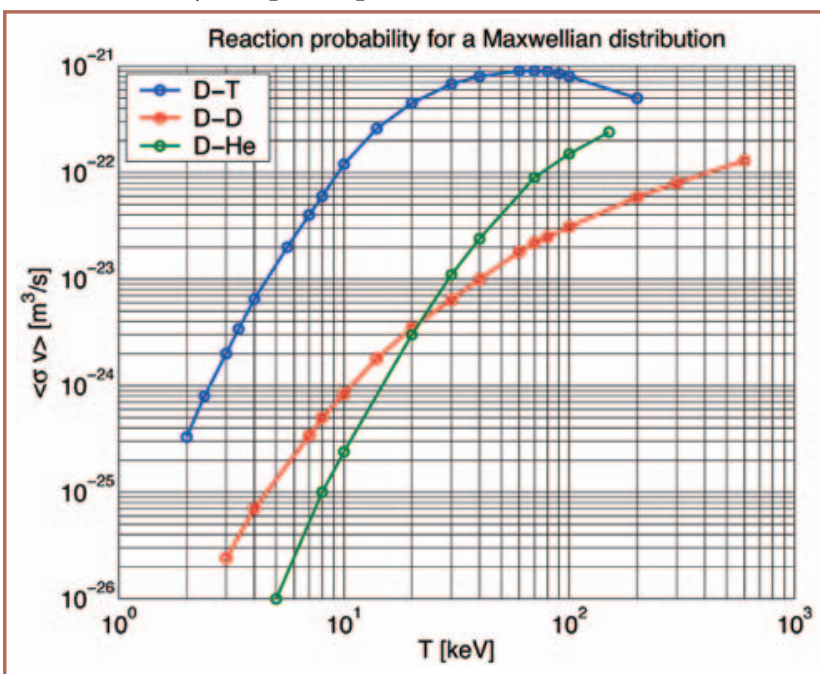

$\Delta$ Fig. 1: Reaction rate coefficients (cross section $\mathrm{x}$ impact velocity averaged over a Maxwellian velocity distribution) for the 3 major fusion reactions as a function of the ion temperature. 


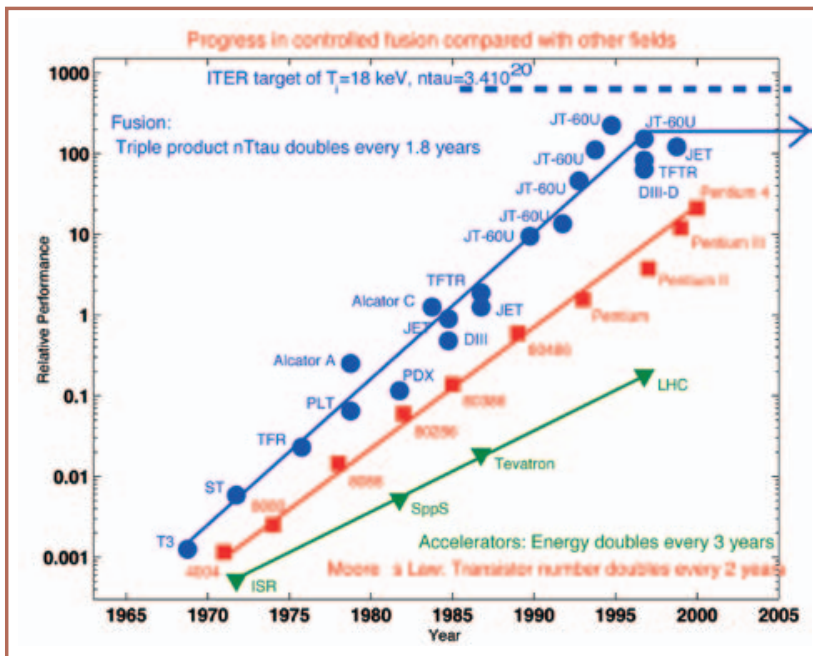

$\Delta$ Fig. 2: The progress of fusion performance figure of merit, $n T \tau$, compared with advances in silicon wafer technology and high energy physics accelerators. The somewhat arbitrary timescale is the date when an experiment was planned for accelerators and performed for tokamaks.

no mechanism for running away. Light nuclei fuse reluctantly because of their electrostatic repulsion. Getting them within the short range of the attractive nuclear forces requires enough energy to overcome the long-range Coulomb potential. As a result the reaction probability or cross section for fusion reactions is significant only for energies well in excess of $10 \mathrm{keV}$. For self-sustained fusion reactions, the fuel must be kept hot by the heat released from its own reactions, implying a confinement scheme for the hot fuel. Criteria for self-sustaining reactions were developed by John Lawson in the early 1950's and defined a minimum value for the product of the fuel density and the characteristic confinement time of the energy in the fuel.

A second difference between fusion and fission reactions is worth recalling. There are only a few fusion reaction channels in the D-T mixture in ITER:

$$
\begin{aligned}
& D^{2}+T^{3} \rightarrow H e^{4}(3.5 \mathrm{MeV})+n(14.1 \mathrm{MeV}) \\
& D^{2}+D^{2} \rightarrow H e^{3}(0.8 \mathrm{MeV})+n(2.5 \mathrm{MeV}) \\
& D^{2}+D^{2} \rightarrow T^{3}(1.0 \mathrm{MeV})+p(3.0 \mathrm{MeV}) \\
& D^{2}+H e^{3} \rightarrow H e^{4}(3.7 \mathrm{MeV})+p(14.7 \mathrm{MeV})
\end{aligned}
$$

On the other hand, fission reactions take place by adding a neutron to create an unstable nucleus, which then splits into a large number of possible final states. This leads to an uncontrolled radioactive mixture of fission end-products, spread over the middle mass range, and remains one of the drawbacks of fission power. These differences lead to the preferred title of "controlled fusion" over "nuclear fusion" to avoid an ill-informed knee-jerk reflex of public opinion.

Fusion reactions only occur at a sufficient rate for useful power production when the nuclei have energies in the range 10$100 \mathrm{keV}$ and can collide almost indefinitely. The cross sections for fusion reactions are much smaller than those for Coulomb collisions between ions, excluding an energetically useful beam-target design of a reactor because most of the beam particles would slow down and thermalise without producing fusion reactions. These conditions are therefore obtained in a confined medium, where the ions have time to thermalise via their electrostatic collisions, giving rise to the original "thermonuclear" label of controlled fusion. The reaction rate coefficients for the most important fusion processes are shown in Figure 1. The Deuterium-Tritium reaction has the largest rate, and is maximum at a temperature of around $60 \mathrm{keV}$ (100 million degrees), at which all the light elements are completely ionised, constituting a globally neutral gas of ions and electrons called a plasma.

The plasma pressure $\mathrm{p} \approx\left(n_{\mathrm{D}}+n_{\mathrm{T}}+n_{\mathrm{e}}\right) \mathrm{kT} \approx 2 n_{\mathrm{e}} \mathrm{kT}$ is limited by MagnetoHydroDynamic (MHD) stability and operation close to the pressure limit provides the highest fusion power for a given temperature. This leads to an optimum operating temperature well below the $60 \mathrm{keV}$ at which $\left\langle\sigma v_{\mathrm{D}-\mathrm{T}}\right\rangle$ is maximum. Beyond this optimum temperature, the increased fusion rate does not compensate the density reduction imposed by the pressure limit.

The fuel has to be isolated from any solid structure to stay hot. Magnetic fields provide the best method of stopping the energetic charged particles from hitting the wall. The dynamics of each single charged particle interacting with a magnetic field and an electric field are simply determined by $\underline{F}=q(\underline{E}+\underline{v} \times \underline{B})$. To a first approximation, the electrons and ions gyrate around magnetic field lines with a radius $\rho_{\mathrm{L}}=\mathrm{v}_{\perp} / \omega_{\mathrm{c}}$ known as the Larmor radius, where $\omega_{c}=\mathrm{qB} / \mathrm{m}$ is the respective cyclotron frequency, rather than cross them. We have to include the radiative energy loss when a particle accelerates in the electric field of an ion, which unfortunately makes the Coulomb collisions between ions and electrons inelastic. We then have to add the nuclear reaction rates, some atomic physics to describe the ionisation equilibrium and radiative losses from collisions with impurity ions, and our knowledge of plasmas should be more or less complete. Why then is this such a complicated field of physics?

The fundamental difficulty is not simply the large number of particles, typically $10^{20}$ per $\mathrm{m}^{3}$ or about $10^{23}$ particles in ITER. The difficulty is that when electrons and ions move, they produce magnetic fields; when electrons and ions drift apart, they create electrical fields. The particles are therefore moving in magnetic and electrical fields generated partly from the outside using magnetic coils (imposed fields) and partly as a result of the movement of all the other particles (self-consistent fields). As a result, we cannot write down a first principles prediction of the collective behaviour of the plasma particles and most importantly, of particle and heat transport through the plasma. A "neoclassical" description of transport resulting only from Coulomb collisions and particle orbits in inhomogenous fields, essentially the imposed fields, was developed more than 30 years ago, but underestimated most of the observed transport processes by 1-2 orders of magnitude. The reason is that low amplitude small scale collective instabilities, with typical wavelengths of a few ion Larmor radii, develop in confined magnetised plasmas, creating turbulence which is responsible for the lion's share of the heat and particle transport out from the hot core of the confined plasma. This turbulence is sustained by the free energy available in the gradients of temperature and number density and causes these gradients to be eroded, unless the transport is compensated by an externally provided source of heat or particles. Despite being the rule, rather than the exception, this form of transport is still referred to as anomalous.

Advances in theory and computing power are still leading to substantial progress in the modelling of anomalous transport, but it is fair to say that a first principles prediction of transport in fusion plasmas is still some way ahead. The major difficulty is associated with the disparity of scales. The ion Larmor radius is typically millimetres, but the distance covered before a fuel ion undergoes a fusion reaction is about $10^{7} \mathrm{~m}$. The electron cyclotron 
frequency is hundreds of $\mathrm{GHz}$, whereas we need to confine the electrons for many seconds. The fundamental problem facing theory is to reconcile these disparate scales in a single model.

\section{Magnetic confinement experiments}

Magnetic confinement experiments have been performed since the 1940's, but it was soon realised that magnetic confinement fusion was not going to be as simple as originally hoped. Devices with open field lines, even with magnetic mirrors, produced by longitudinal field gradients, proved to be too lossy. Today all magnetic field configurations considered to have potential for fusion have a set of nested magnetic flux surfaces with twisted field lines produced by the combination of magnetic fields. The most advanced and one of the simpler ones is the tokamak, which was developed in the former Soviet Union in the 1960's and has the symmetry of a torus. The toroidal (parallel to the torus "tyre") magnetic field of the tokamak is produced by external coils like a circular solenoid, while the second, slightly weaker field necessary for twisting the field lines, is produced by a current flowing through the plasma itself in the same toroidal direction combined with the field from toroidal coils external to the plasma. In most tokamaks this current, which reached 7 million amps in JET and will be up to 17 million amps in ITER, is induced in the plasma by a transformer coil. Driving the plasma current non-inductively using particle beams or radio-frequency waves has been demonstrated in many devices and will be used in ITER.

Many tokamaks were built throughout the industrial world after the Russian successes in the 1960's. 5 million Euros get you a rather nice plasma device with a temperature around $1 \mathrm{keV}$, but not nearly hot enough, nor well enough confined, for fusion power. The seventies and eighties saw the construction of generations of successively larger tokamaks, with the three largest, JET (Europe), JT-60 (Japan) and TFTR (USA) completed in the early 1980 's. These devices have already achieved the plasma densities and temperatures required for a fusion reactor, but their confinement time, the ratio of the plasma kinetic energy to the heating power, is still an order of magnitude below that required for selfsustained burning of the plasma fuel. Confinement improves as the plasma cross section increases, roughly as expected from diffusion and also increases with the plasma current. The latter is limited by MHD stability and scales linearly with the plasma minor radius and toroidal magnetic field.

Figure 2 illustrates the progress made in designing and operating tokamaks, compared with the rapid progress of accelerator energies and chip densities, the well-known Moore's law. Tokamaks have increased their performance, expressed by the product of temperature, density and confinement time, by 6 orders of magnitude in the thirty years between the first experiments and the time of the JET deuterium-tritium experiments of the last decade of the $20^{\text {th }}$ century. To be sure, as size and performance increased, so did the costs and the manpower requirements, necessitating wide national and supranational funding sources and collaborations as in the case of JET, which was constructed by the members of what then was the European Community, with Swiss and Swedish participation.

During this period, a large number of tokamaks investigated different means of heating the plasma, increasing the delivered power from the $10 \mathrm{~kW}$ level to the $20 \mathrm{MW}$ level in present devices. At the same time, our skills in measuring the properties of the hot plasma were refined, simple "diagnostics" being replaced by huge arrays of detectors to measure the radiation and particle emission from the plasma, or to send particle or electromagnetic beams into the plasma. The advances in diagnostic techniques led to a parallel development of interpretative analysis and modelling codes. This work continues.

\section{The next step}

The last 25 years of experimental work in a large number of different tokamaks has provided us with an extensive database on fusion plasmas and most importantly, on the scaling of confinement with plasma size [2]. Projections of the performance of next step devices such as ITER and fusion reactor studies are largely based on empirical and semi-empirical scaling laws derived from this work and on theoretical models benchmarked against the experimental database. Figure 3 shows the experimental confinement time versus the empirically regressed one, together with the extrapolation for ITER.

Choosing the device to construct as a next step has been a question of optimising what will be learned from the project and how useful this information will be in going ahead in the future. In the mid-1980's, Europe was busy preparing a large project (Next European Tokamak, NET) which stalled when the multi-national ITER project including Europe, the then Soviet Union, Japan and the United States, was launched as a conceptual design activity in 1988. ITER appeared more attractive, splitting the costs. In 1992 the partners agreed to progress towards the engineering stage. Six years of international collaborative work within the framework of the ITER Engineering Design Agreement culminated in the approval by the ITER Council in June 1998 of the ITER Final Design Report, Cost Review and Safety Analysis. This provided a comprehensive design of a fusion reactor based on well established physics and technology. Its design fulfilled the overall programmatic objective of ITER - to demonstrate the scientific and technological feasibility of fusion energy for peaceful purposes.

Unfortunately, ITER itself then stalled in 1998, when countries baulked at the cost and a "reduced objectives" ITER was designed by a tripartite collaboration of the European Union, Japan and

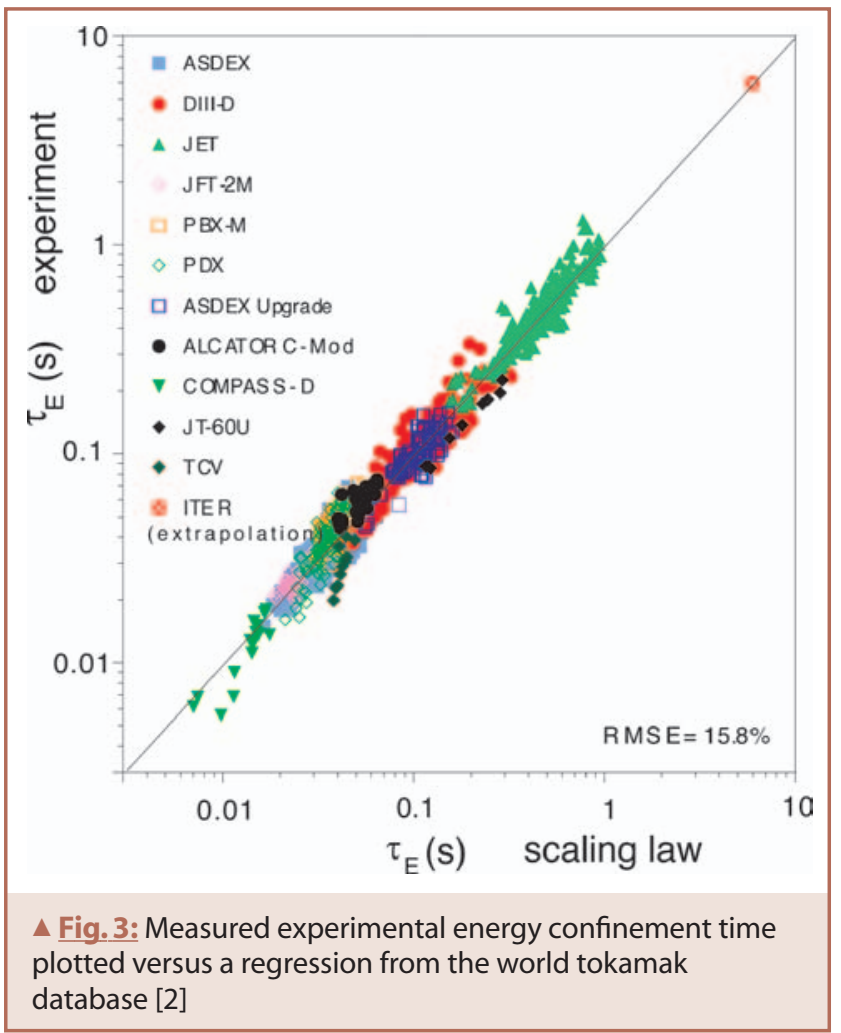


Russia, with the aim of cutting the capital investment by half, while maintaining all of the key physics objectives and as many of the technology objectives as possible, especially the programmatic objective that ITER should be a single step between the present experiments and a fusion power reactor. This redesign led to a reduced size, reduced performance, reduced margin design, delivered to the remaining parties in 2001, which ironically but not surprisingly, is not unlike the size of the NET design of the 1980 's. Reference [3] provides a wealth of design information. The reduction in size also increased the pressure on researchers working on tokamak experiments worldwide to find ways of subtly enhancing the performance beyond the baseline reflected in the scaling laws.

The extrapolation of the experimentally measured energy confinement time in existing tokamaks towards ITER is illustrated in Figure 3, and is considered to be robust because ITER does not differ any more in size from JET and JT-60 than these devices themselves differ from several currently operating medium size devices. Yet ITER will enter unexplored territory. The relevant physics variables are not the engineering parameters of the device, but dimensionless parameters such as the pitch of the field lines, the ratio of plasma pressure to the magnetic pressure $\left(\mathrm{B}^{2} / 2 \mu_{0}\right)$, the ratio of the Larmor radius to the machine radius and the ratio of the Coulomb collision frequency to the frequency of the periodic drift motion in the inhomogenous field. The combination of the values for the two latter parameters (key parameters for anomalous transport), as required for a reactor, cannot be achieved in current devices and must be assessed by experiments in a reactor sized device.

Extrapolation of other key plasma parameters and present tokamak operating procedures to ITER also has uncertainties, because the reactor plasma conditions cannot be fully emulated in present day devices. The mission of ITER will be to demonstrate that all the physics issues, and most of the technology issues, that fusion reactors will be facing can be dealt with in an integrated way.

These issues include the effects of the alpha particle population produced by the fusion reactions on the plasma. After birth at $3.5 \mathrm{MeV}$, the alphas slow down, transferring their energy and momentum to the thermal particles, mostly to the electrons. In ITER they are expected to provide more than $2 / 3$ of the heating power necessary to sustain the plasma, corresponding to the target fusion gain, $\mathrm{Q} \sim 10$, where $\mathrm{Q}$ is the ratio of the fusion power to the auxiliary heating power. Energetic particles are known to stabilise certain usually benign instabilities in the plasma core, as well as to produce new kinds of plasma instabilities, which may enhance transport, especially that of the alpha particles, and thereby reduce the fusion power. The transport of fuel to the hot core, where most of the fusion power is released, and the transport of the resulting helium ash away from it, is also an issue. If particle transport is too slow compared with heat transport, fusion output is reduced as the fuel slowly poisons itself with helium, increasing the radiated power loss and diluting the fuel.

Other issues are linked to the 400-1000 second duration of the discharges in ITER and the necessity of actively cooling the plasma facing vessel components. The very nature, magnitude and distribution of the heat load is particularly difficult to extrapolate because of the wide variety of behaviour observed in existing devices. The last closed flux surface (LCFS), defining the confined plasma, is not in direct contact with a material wall. Beyond the LCFS a set of open field lines channel the heat and particles from the plasma towards a target area called a divertor, seen as a notch in the lower part of the vacuum vessel in Figure 4. On the way, the majority of the heat is lost as radiation, cooling the edge plasma. However part of the power flowing to the divertor arrives as millisecond bursts of energy, caused by MHD instabilities at the plasma edge, which can cause accelerated erosion of the plasma facing components, as well as contaminating the plasma with impurities and diluting the fuel. These bursts vary from insignificant to excessive in current devices, which are developing countermeasures. The general control of plasma-wall interactions needs to be demonstrated in ITER before the road is cleared towards a reactor. The avoidance and handling of potentially damaging sudden terminations of the plasma current and

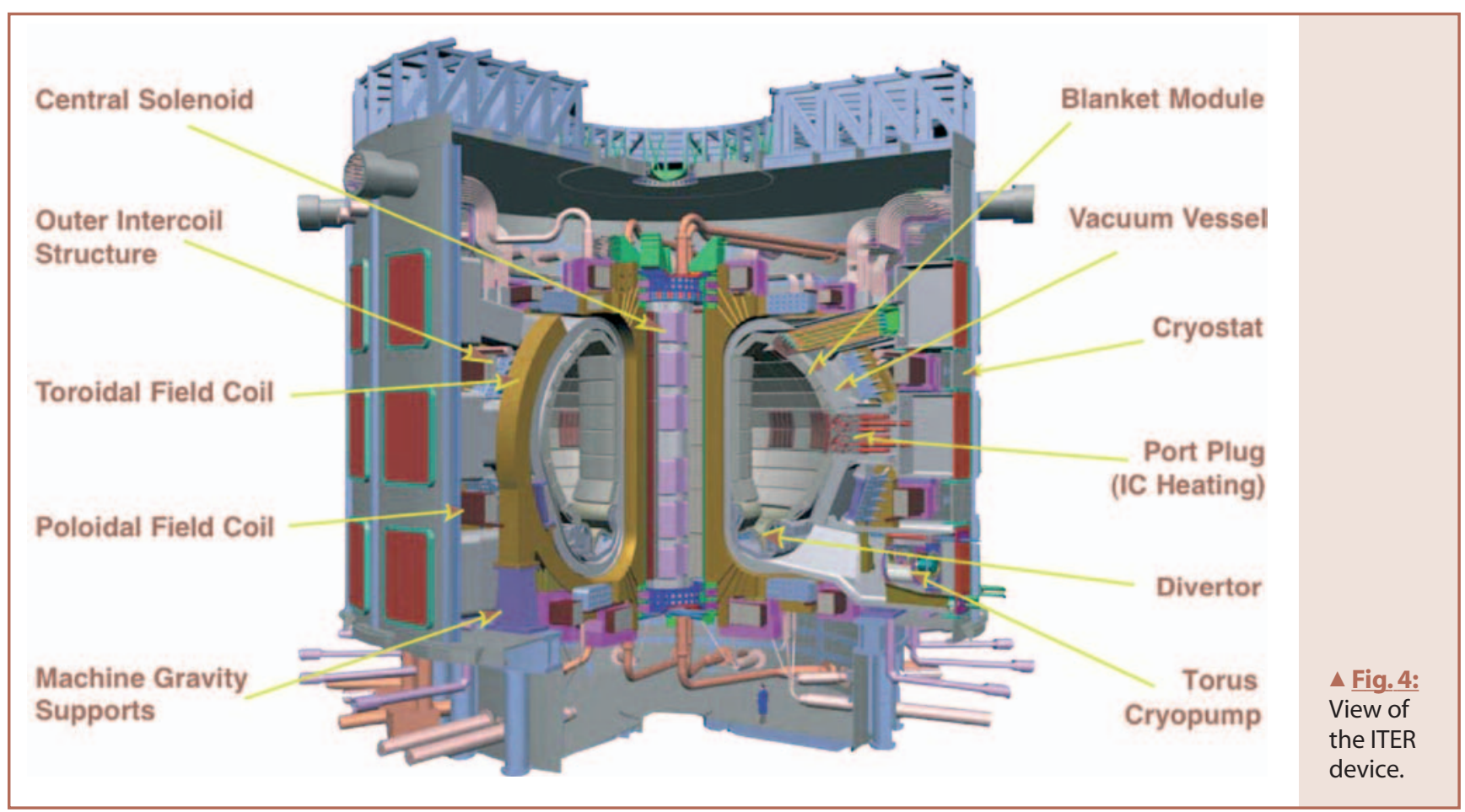


confinement, known as plasma disruptions, which can lead to large $i \times \underline{B}$ forces on the in-vessel components, will also need to be rehearsed in ITER.

ITER is a formidable technological challenge that must be met before physics investigations can even begin. ITER contains 400 tonnes of superconducting $\mathrm{Nb}_{3} \mathrm{Sn}$ and $\mathrm{NbTi}$ within its 48 coils and will have to demonstrate 2 decades of superconducting magnet operation. It must demonstrate that the auxiliary heating methods developed over the past three decades allow us to heat the plasma up to the point where alpha particle heating takes over. It must demonstrate the handling of high heat fluxes on the plasma facing components, especially in the divertor. It must demonstrate the capability of pumping the helium ash out of the vessel, tritium handling and fuelling. Reactors will ultimately have to produce their own tritium in breeding blankets by making use of the $\mathrm{n}+\mathrm{Li}^{7} \rightarrow \mathrm{He}^{4}+\mathrm{T}+\mathrm{n}$ or the $\mathrm{n}+\mathrm{Li}^{6} \rightarrow \mathrm{He}^{4}+\mathrm{T}$ reactions, where the reacting neutrons are produced by the fusion reactions in the plasma. ITER must perform functional tests of concepts of tritium breeding blankets required for a reactor.

There remains one important technology issue that ITER is not designed to deal with, which concerns the testing of radiation resilient low activation materials that will be required for a reactor. Due to its low average operational duty cycle as a research installation, neutron induced damage in the structural materials inside the vessel, built mainly from conventional austenitic steel, will not be an issue in ITER, nor will ITER be a suitable test environment for the most stringent requirements of materials developers. The development of materials suitable for fusion reactors requires an effort parallel to the construction and operation of ITER and will involve the construction of the International Fusion Material Irradiation Facility IFMIF, a beam/target neutron source, which will generate continuous $14 \mathrm{MeV}$ neutron loads comparable to those of a reactor in a small test volume for material samples.

ITER is not an end in itself. It is the bridge between the devices built in the 1980's and a demonstration power plant. The essential role of ITER is to validate the tokamak fusion concept as a viable approach to power production and to develop the reactor technologies needed for any fusion power plant. The endeavour will also spur valuable fundamental advances in physics and technology. In view of the promise of fusion and of the necessity of developing new sources of energy that are environmentally acceptable and close to inexhaustible, we believe that ITER is a step well worth taking. The members of the ITER design teams have steadfastly brought this project to the point of fruition all through the difficult times of the successive design phases. Let their effort be crowned by success.

\section{About the authors}

Jo Lister and Henri Weisen, of British and Luxembourg origins, are involved in experimental tokamak research at the CRPP-EPFL in Lausanne, Switzerland. Jo Lister is currently chairman of the EPS Plasma Physics Division. Both authors are engaged in extensive international collaborations and are fervent supporters of a positive decision to go ahead with ITER.

\section{References}

[1] www.jet.efda.org

[2] “ITER Physics Basis", Nuclear Fusion 39 (1999), by the ITER Physics Basis Editors, ITER Physics Expert Groups, ITER Joint Central Team and Physics Integration Unit

[3] www.iter.org

\section{Astronomy and Astrobiology}

David Field, Department of Physics and Astronomy, University of Aarhus, Ny Munkegade, Building 520, 8000 Aarhus C

$A$ strobiology is the study of the origin of life on Earth, and whether life may exist elsewhere in the Universe. This article will seek to convey some of the new discoveries in Astronomy which relate to Astrobiology and which have energised the field. These discoveries are little isolated illuminated patches and it is difficult to link them together into a coherent picture. More questions are raised than answered, but this is only as it should be; we do not understand the origin of life, but we are beginning to assemble pieces of the puzzle.

\section{An overview}

For the range of life as we know it, we need an abundance of the elements oxygen, carbon, hydrogen, nitrogen and also traces of many others, such as magnesium, silicon, iron, aluminium, sodium, potassium, chromium, manganese, phosphorous and more, even including tungsten. All heavy elements necessary for life have been created in the interior of stars in the 13,500 million years since the Big Bang. The massive first star in the Universe [1] was formed roughly one million years after the Big Bang and exploded as a supernova. In this explosion, small amounts of elements heavier than $\mathrm{H}$ (and $\mathrm{He}$ ) were made from nuclear reactions at the very high temperatures of the explosion.

The Universe reassembled, so to speak, after this first destructive event to make a generation of new stars. Perhaps 100 million years later the first galaxies formed. Their component stars were quite unlike our Sun. They were again massive, and exploded as supernovae, spreading heavy elements throughout their host galaxies. This created a medium enriched in heavier elements between the stars, an "interstellar medium". A new generation of stars formed in this interstellar medium in turbulent knots of gas with high local density, and ultimately, as for all stars, through the overwhelming force of gravitational attraction.

Recent simulations suggest that this new generation of stars contained stars was of similar mass to the Sun [2]. These stars would have been much longer-lived than earlier stars. However biology was not yet feasible since the abundance of heavy elements was still very low, for example 100 times below that of the Milky Way. Sufficient raw materials for rocky planet formation, let alone life, were still lacking. Further generations of stars lived and died, each enriching the medium between the stars with heavy elements. As time has gone by, $1.7 \%$ of $\mathrm{H}$ atoms created in the Big Bang have been converted into elements heavier than He.

Nine thousand million years after the Big Bang, in one of the spiral arms of the Milky Way, a Galaxy among more than $10^{11}$ others, a particular cloud of gas - the "pre-solar nebula" - containing the elements necessary for life, began to undergo gravitational collapse and formed the Sun. There was a sufficient abundance of elements such as oxygen, silicon, iron, magnesium, aluminium and sulphur to form rocky planets. At this juncture, we enter a realm in which our ignorance is paramount. After a few hundred million years, single cell organisms appear to have formed, though how this happened from the bare chemical ingredients of 